\title{
On the Evolution of Coordinated Multi-Point (CoMP) Transmission in LTE-Advanced
}

\author{
Md. Shipon Ali \\ Senior System Engineer, Grameenphone Ltd., Dhaka, Bangladesh \\ shipon@grameenphone.com
}

\begin{abstract}
Inter-cell interference is a serious issue in Long Term Evolution (LTE/LTE-Advanced) system. Coordinated Multi-Point (CoMP) operation has been considered as a powerful technology targeted for LTE-A standard to reduce interference, improve spectrum efficiency, increase data throughput and enhance effective coverage area, in particular for cell-edge users, by exploiting the interference signals from different transmission points. In this context, this paper provides a holistic overview of CoMP operation in LTE-A system. Here, we consider the downlink operation, e.g. CoMP transmission, as it is more convenient to explain the system effectively. The technical operation challenges, advantages and operation complexities of different CoMP schemes, CoMP architectures and CoMP sets are described in details in terms of backhaul traffic, synchronization effect and feedback design. Moreover, this article provides clear evidence of CoMP benefits in terms of throughput gain and compares the results with no-CoMP scenario. In addition, it is intended for a wide range of readers as it covers the topic from basics to advanced aspects.
\end{abstract}

Keywords: LTE-Advanced, CoMP, HetNets, Inter-cell interference

\section{Introduction}

In last couple of years the wireless industry has been experiencing huge increasing demand for data traffic over cellular network, and the development of advanced high-speed user handsets, e.g. smart-phones and tablets, further expedite this demand. Users nowadays expect to high-speed data connectivity at anytime from anywhere. The evolution of Long Term Evolution (LTE) [1], commonly known as 4G, has been brought the high speed wireless technology for mobile users. Orthogonal Frequency Division Multiple Access (OFDMA) used in LTE potentially avoids co-channel interference due to its inherent feature [2]. However, inter-cell interference caused by neighboring Base Stations (BSs) has been marked as one of the most severe problem towards the deployment of LTE technology as it can significantly deteriorate the performance of cell-side User Equipments (UEs) [2].

In spite of high speed wireless connectivity of LTE, the traffic demand still increasing and the traditional homogeneous networks fall short to satisfy these demands. To meet these requirements, recently, a new framework called heterogeneous networks (HetNets) has emerged as a flexible and cost-effective solution [3-6]. It is realized by overlaying low-power access points such as relay node, picocell BS, femtocell BS and Remote Radio Head (RRH) in the coverage area of macrocell. However, this overlaying of macro and low-power cells with different transmission powers enlarges the inter-cell interference zone. To mitigate this problem Third Generation Partnership project (3GPP) released LTE-Advance along with CoMP technology [7-10, 12-14]. CoMP, also known as network multiple-input multipleoutput (MIMO), is one of the most promising techniques for improving the data transmission 
efficiency of wireless cellular networks. The main idea of a CoMP network is to allow geographically separated base stations (BSs) to cooperate in serving the users. CoMP schemes allow interference mitigation through joint/coordinate transmission/scheduling from multiple BSs, but at the cost of increased complexity and signaling overhead [11, 16].

The rest of the paper is organised as follows: section 2 presents the motivation towards this work and also mentions related major works that have been previously performed in this regard. A basic CoMP principle is discussed in section 3. The detail description of various CoMP techniques, CoMP implementation architectures and CoMP sets are presented in section 4 and upwards, respectively. The performance evaluation of CoMP with different network scenarios is covered in section 7. Finally, section 8 contains the concluding part along the potential challenges of implementations.

\section{Motivation And Related Works}

The performance of LTE/LTE-A is largely affected by the inter-cell interferences of neighboring BSs. In heterogeneous implementation this situation becomes more severe as more UEs experience interference from other BSs due to creates more cell boundaries. As a result interference management is crucial to ensure proper QoS. In this regards, CoMP technology is the most potential solution where interfere signals from other BSs are exploited to increase overall throughput.

CoMP technology in LTE/LTE-A is studied in several literatures [12-16]. Authors of [12] discussed CoMP in the context of heterogeneous implementation, where they examined the applications of cooperative relaying schemes in LTE-advanced systems. In [13] authors studied the feasibility of COMP in two field test-beds with multiple sites and different backhaul solutions between the sites. Similar survey with the feasibility test to practical implementation is carried out in [14]. Authors of both survey [13, 14] examined technical issues in terms of backhaul traffic, synchronization and feedback design. Moreover, in context of multi-cell interference a survey report is present in [15] where authors correlated and compared CoMP with other techniques, namely static ICIC and dynamic ICIC. Furthermore, the impacts of control channel in which the signaling is performed among CoMP set BSs and other unit is studied in [16] in respect to unreliable backhaul network.

\section{Basic CoMP Principle}

The fundamental principle of CoMP [8-10], is utilized multiple transmit and receive antennas from multiple antenna site locations, which may or may not belong to the same physical cell, to enhance the received signal quality as well as to reduce interference, improve spectrum efficiency and enhance effective coverage area by exploiting the co-channel interferences. CoMP mainly has been targeted to improve cell-edge UE experience, but regardless the location it also use to enhance system through to UEs those experience strong signals of different BSs/cells. CoMP mainly categorized as inter-site CoMP and intra-site CoMP. In inter-site CoMP, the coordination is performed between BSs located at separated geographical areas. On the other hand, intra-site CoMP enables the coordination between sectors of the same BS, where the coordination is performed through multiple Antenna Units (AUs) that allow the coordination between the sectors. Figure 1 illustrates both CoMP categories. 


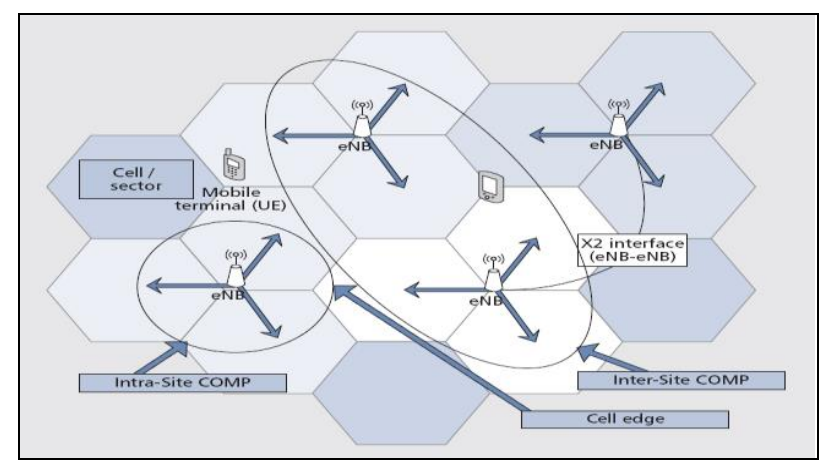

Figure 1. Basic CoMP Technology; Inter-site CoMP and intra-site CoMP

There are several techniques available for CoMP technology where most approaches require some scheduling information regarding the UEs data at the different BSs that must be shared among them. This sharing results increased complexity and signaling overhead on network, especially on backhaul network which is commonly known as X2 interface in LTE/LTE-A. In downlink CoMP, UEs report Channel State Information (CSI) to BSs based on the experienced SINR of respective BSs. This CSI report is sent in uplink transmission. In LTE MIMO systems, Linear Precoding [17-18] is used as a method to improve the performance in downlink and to decrease the signaling load between the cells. Therefore, in CoMP system, to indicate the possible channel states in both transmitter and receiver a set of pre-defined precoding matrices is used. The Precoding Matrix Index (PMI), which is defined as the index to the preferred matrix within in a codebook matrix, is reported by UE together with Channel Quality Indicator (CQI) and MIMO Rank Indicator (RI) [18]. CQI and PMI are parameters determined by UE based on pilot measurements and UEs send to their serving BS. Moreover, RI indicates the MIMO ranking i.e. the number of data streams to be transmitted in parallel for the next transmission over the MIMO channel [19]. These parameters are transmitted in a quantized manner to BS so as to reduce signaling overhead. By coordinating and combining signals from multiple antennas, CoMP makes it possible for UEs to enjoy consistent performance and quality when they access and share videos, photos and other high bandwidth services whether they are close to the center of an LTE-A cell or at its outer edges.

\section{Different CoMP Techniques}

In downlink scenarios, CoMP initially classified into two main schemes, namely Coordinated Scheduling/Beam-Forming (CS/CB) and Joint Processing (JP). These schemes also furthered sub-categorized. In this section the whole schemes is present.

\subsection{Coordinated Scheduling/Beam-Forming (CS/CB)}

In CS/CB CoMP [20], the data packets that need to be conveyed to a UE terminal are available at and transmitted from only one BS in the CoMP cooperating set, whereas user scheduling and beamforming decisions are made dynamically after the coordination among all points in the cooperating set. Semi-Static Point Selection (SSPS) is used to make the transmission decisions. CS/CB provides fast and strict coordination, but meanwhile it uses the MIMO antenna capabilities through Beamforming in a coordinated manner. Here, the best serving set of users are selected for the construction of the transmitter beams based on their geographical positioning. The coordinated generation of beams and more specifically the beam-to-resources selection manages to reduce interference to other neighboring users, while 
increasing the target users signal strength. Different approaches jointly combining CS and CB have been studied in LTE-Advanced, which can be classified by increasing order of complexity and requirements in terms of CSI feedback and CSI sharing. In this way, CS/CB can be categorized to Coordinated Beam-Switching (CBS-CoMP) and Coordinated Scheduling (CS-CoMP) schemes.

4.1.1 Coordinated Beam-Switching CoMP (CBS-CoMP): CBS-CoMP is technique in which each cell determines a sequence of beams over which it continuously cycles. Most proposals in this schemes focus on distributed coordination between the cell sites. A low complexity scheme is proposed allowing the coordination of two sectors with direct communication is proposed in [21]. It is a point-to-point coordination, where sectors agree on the way resource allocation is handled for all possible conflicting beam pairs. However, this proposal is limited to perform coordination only for two BS. On the other hand, authors in $[22,23]$ propose a cyclic beam switching scheme that assumes a fixed beam cycled period for all cell sites. In this proposal, the UEs group based on their geographical location are experienced a set of interference fluctuations which is predictable to the schedule. However, based on the CSI reports for each frame, each BS cycles through a set of preferred beams where the pattern of this cycle depends on the geographical location and the QoS parameters. Consequently, the coordination of BSs is required only in the frame synchronization in the time domain and the decision of a common cycle period. In the same philosophy, in [24], an autonomous beam coordination scheme is proposed that targets predicting interference fluctuations by BSs through beam coordination in frequency domain. Based on the CSI reports, the cooperating BSs allocate beams-to-resources autonomously for a group of users in dynamic manner. For the inter-site interference mitigation, each cell maps the possible gains that it could achieve by changing its allocation using the corresponding probability of a change to occur.

On the other hand, in [25] a centralised coordination of CBS-CoMP is proposed, where a BS is acts as a central unit in which a Master Scheduler is present, and jointly schedules multiple cells in a centralized manner. Based on feedback reports, UEs divide in two groups: Collision Avoidance (CA) UEs those face high interference and assume to be cell-edge users, and Non-CA UEs those face lower interference and assume to be cell-central users. UEs of both groups report their preferred PMI and CQI to their serving cell after measuring all PMIs and CQIs of interfering cells. Consequently, the master scheduler picks the highest priority serving sets of UEs for each cell and allocates beams to each set upon highest sum capacity.

4.1.2 Coordinated Switching CoMP (CS-CoMP): CS-CoMP is one of the main CoMP schemes that perform cooperation by enabling coordination of multi-cell Precoding Matrix Index (PMI) between cooperating BSs [27-28]. In this case, the BSs can directly perform the coordination of preferred UE's PMIs without any centralized scheduler. UEs recommend a set of PMIs of other BSs of the CoMP set instead of restricting the use of beams-to-resource. There are two different strategies of PMI coordination as defined in [27] based on how feedback PMI sets are selected by BSs: Good-PMI feedback that is chosen when cell-edge UEs report a set of least-interfering PMIs to the BS, and Bad-PMI feedback that allows a BS to mitigate ICI by restricting strongly interfering PMIs. In this case, BS does not require adding more scheduling restrictions to its neighbor BSs. However, UEs reports their preferred PMIs to serving BS and also expected CQI improvements in case the recommended PMIs are used. The BS then exchanges signaling information with interfering BSs that indicates the PMI restrictions or preferences as per PMI policy, and then decides the precoding vectors and matrices for the UE it serves. 


\subsection{Joint Processing CoMP}

JP-CoMP [11-13] is the most advanced CoMP schemes which basically investigate to improve spectral efficiency, in particular cell-edge user spectral efficiency. Here, data for a $\mathrm{UE}$ is available at more than one point in CoMP set for same time-frequency resource; by default at all points [1]. In terms of cooperation mechanism JP-CoMP is subclassified into two main groups, namely Joint Transmission CoMP (JT-CoMP) and Dynamic Point Selection CoMP (DPS-CoMP). Both of are discussed in below subsections.

4.2.1 Joint Transmission CoMP (JT-CoMP): The most powerful CoMP scheme is JTCoMP [13]-[14] in which a UE data is simultaneously process and transmitted from multiple cooperating BSs. In heterogeneous scenario and dense small cell network with low power node, UEs experience significant signal strength simultaneously from multiple BSs. Hence JT-CoMP is more attracting feature to improve spectral efficiency as well as average throughput in this case. However, according to CSI report and transmission mode JT-CoMP is further classified into two types: Coherent JT-CoMP and non-coherent JT-CoMP. In coherent JT-CoMP, UE sends CSI report consisting preferred PMI, expected CQI and wideband RI to all cooperating BSs. Therefore, multi-cell precoding cooperation is performed between the BSs and resulting a unique pre-coder for all transmission points of the CoMP set. Thereafter, all BSs jointly process PMI-to-resource allocation and simultaneously transmit to UE. As a result a quite high combination gain signal is obtained. Coherent JT-CoMP is also known as closed loop JT-CoMP as it performs closed loop MIMO operation. In spite of significant performance gain, high signaling overhead on backhaul network, particularly on $\mathrm{X} 2$ interface, UE movements and timing mismatch are the major drawbacks of coherent JTCoMP [29].

On the other hand, Non-coherent JT-CoMP may use techniques like Single Frequency Network (SFN) or Cyclic Delay Diversity (CDD) schemes, which targets diversity gains and increased transmit power to the UE. As a result it is less sensitive to UE's movement and cooperatively low overhead signaling on backhaul network. In non-coherent JT-CoMP, UE's reports CSI to all cooperating BSs that consists of sub-band CQI and the wideband RI. Then single cell precoding is used autonomously by each BSs and only CQI information is exchanged between the BSs. Finally, each BS individually transmits data to UEs in downlink and a non-coherent combination is performed at receiver terminals.

4.2.2 Dynamic Point Selection CoMP (DPS-CoMP): Dynamic Point Selection (DPS) is a special JP-CoMP scheme in which UE's have the opportunity to reselect serving BS based on the highest received SINR and minimum path loss. Unlike CS-CoMP the UE's data is available at all cooperating BSs, but the UE dynamically selects the best serving BS for the next frame and reports to all cooperating BSs of CoMP set. Then, the selected new serving BS notifies the other cooperating BSs over X2 interface and sets to mute them for the resources that this UE is going to use. Hence, the data transmission is occurred only by one BS at a time. In this process, the proper utilization of available spectrum is not possible. However, an enhanced DPS algorithm with improved performance that enables an adaptive muting mode selection and power control is proposed in [31].

\section{CoMP Set}

In downlink transmission, CoMP set generally defined as the group of BSs within a geographic area those are directly or indirectly participating in data transmission to a UE. The UE may or may not know about this set. The direct participation BSs are those actually 
transmitting data, for example all cooperating BSs of JT-CoMP, serving BS of CS-CoMP and DPS-CoMP etc. On the other hand, the indirect points are those involved in cooperative decision making for user scheduling/beamforming in the time and frequency domains, like except serving cell other cooperating BSs of CS-CoMP and DPS-CoMP etc. By default all BSs under a CoMP set perform cooperation during data transmission to UEs, but it is possible only a part of these BSs to coordinate for a cell-edge UE. There are several proposals in 3GPP LTE/LTE-A that defines CoMP set according to different deployment scenarios. The main three approaches are: Network Centric, UE Specific and Network-centric UE-assisted [32, 33].

\subsection{Network Centric CoMP Set}

Based on the neighboring BS signal and planning consideration BSs are grouped in clusters at network level. Each cluster is called a CoMP set in terms of network point of view and defined for all UEs under the BSs of that cluster. In this approach a portion of BSs under the cluster take parts in transmission/scheduling decision for individual UE. Though it is a simple technique but can perform effectively if all strong interference BSs is grouped together into a cluster. However, these kinds of CoMP set resultant large cluster size which may increase the signaling overhead in uplink and consume excessive power from UE terminal.

\subsection{UE-Centric CoMP Set}

In opposite to network-centric COMP set, UE-centric approach forms based on the strongest interferer signals experienced by UE. It is a semi-dynamic process in which the CoMP size can be modified depends on UE feedback, and largely mitigate high signaling overhead. All the cooperating BSs under UE-centric CoMP set actively participate in transmission/scheduling decisions. However, to select appropriate set-size and perform multicell scheduling is more challenging in UE-centric CoMP.

\subsection{Network-centric and UE-assisted CoMP set}

In this approach, the advantages of both of network-centric and UE-centric CoMP set are merged together to form an optimal set. Here, the network pre-defines a group of CoMP patterns and the selection of the suitable scheme is based on the feedback information from UE. This process greatly optimizes the signaling overload and operational complexity.

However, for all three of above CoMP clustering strategies the major challenge is the choice of the optimal number of cooperative BSs in a CoMP set. In this regards, authors of [34] propose a dynamic clustering algorithm to cluster BSs into CoMP sets using the instantaneous channel state information received by the UE. For every time slot, each UE chooses the BSs cooperation set that jointly transmits to ensure maximum throughput to UEs. On the other hand, to achieve high spectral efficiency gains while keeping the backhaul signaling reasonable [35] proposed a dynamic selection of CoMP set with optimal BSs number.

\section{CoMP Architecture}

CoMP architectures deal with the fundamental way of implementation in which the BSs of CoMP set are coordinated to handle interference resulting improve throughput. Basically, there are three different types of CoMP architectures available, namely centralised architecture, semi-distributed architecture and fully-distributed architecture. These architectures are discussed in following subsections. 


\subsection{Centralised CoMP Architecture}

The centralised architecture [37] is depicted in Figure 2. In this architecture a Central Unit $(\mathrm{CU})$ is used to perform coordination between the cooperating BSs to handle interference i.e., enhance throughput. Under this architecture, each BS gets local CSI report directly from serving UE and then forwards to the $\mathrm{CU}$, which is supposed to be located in core network [38]. Based on the gathered system channel matrix, the CU constructs the precoding matrix for JT-CoMP scheme or designs scheduling decisions for the CS-CoMP scheme. Then the designed precoding/scheduling decisions are forwarded to cooperating BSs. Finally, the BSs of CoMP set transmit precoding/scheduling information to their serving UEs as per CoMP techniques. But, the major drawback of this architecture is the high overhead signaling and the stringent latency requirements. A potential but expensive solution to this problem is connecting central unit and coordinated BSs via optical fibers [39].

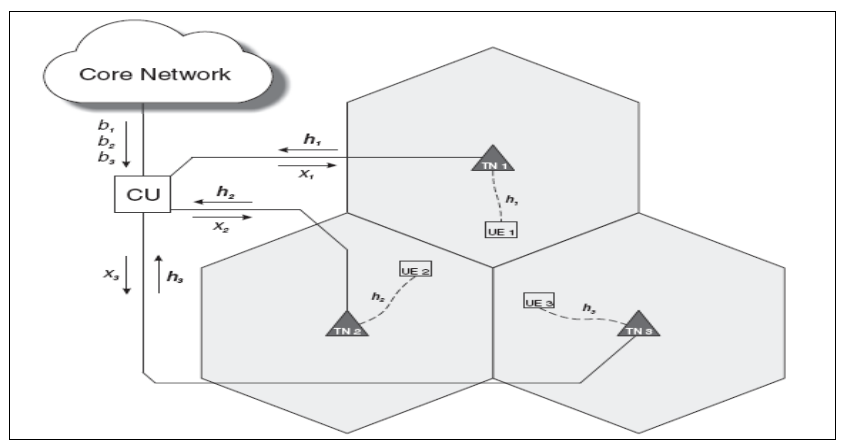

Figure 2. Basic principle of Centralised CoMP Architecture

\subsection{Semi-Distributed CoMP Architecture}

In semi-distributed architecture [40], each BS receives local CSI reports directly from its serving UE and also gets $N-1$ non-local CSI vectors from other $N-1$ coordinated BSs (assumed that CoMP set consists of N number of BSs) via X2 interface. However, based on the all gathered CSI reports, each cooperating BS acts as a CU, independently designs its own precoding matrix or scheduling decisions and also power allocation vector and send to UE $[41,42]$. Therefore, transmission decisions are locally applied to the UE. The data blocks sent from the core network to BS will contain all data symbols for the scheduled UEs in the cluster. This technique greatly reduce backhaul load but required a full mesh reliable connectivity between the cooperating BSs which is considered a big challenge. Figure 3 illustrates the semi-distributed CoMP principle.

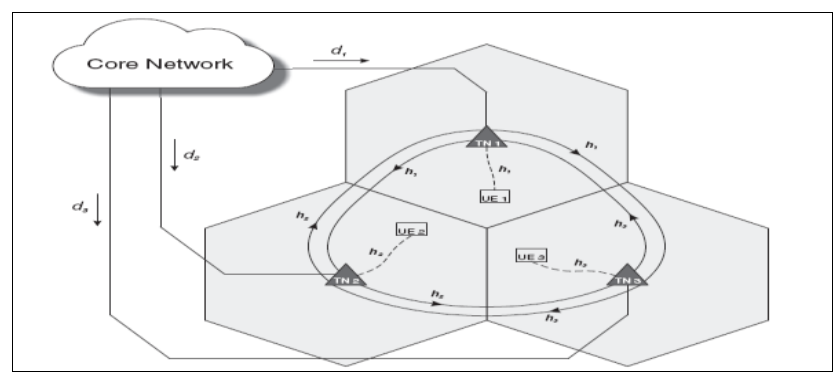

Figure 3. Basic principle of Semi-Distributed CoMP Architecture 


\subsection{Fully-Distributed CoMP Architecture}

Unlike the other two architectures, in fully-distributed CoMP architecture [43], every UE broadcasts their CSI reports to their all cooperated BSs via radio access link. Based on the gathered CSI each BS independently constructs the precoding matrix for JT-CoMP scheme or designs scheduling decisions for the CS-CoMP scheme. The principle of fully-distributed CoMP is illustrated in Figure 4.

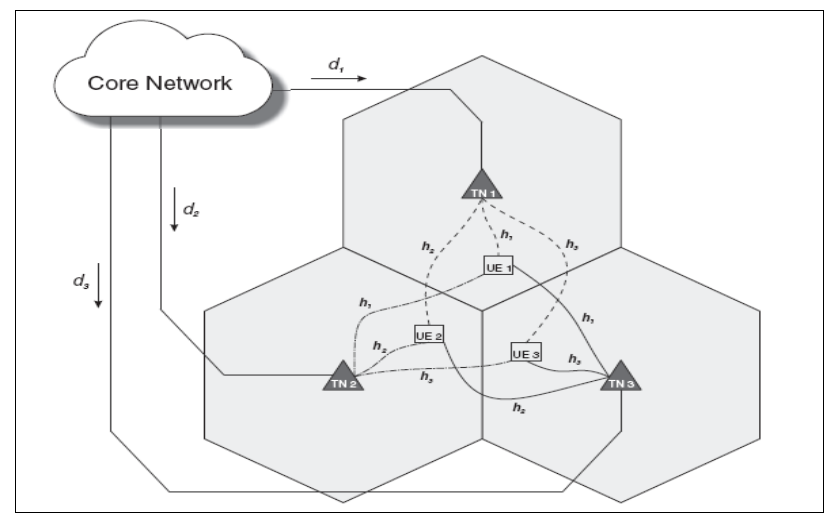

Figure 4. Basic Principle of Fully-Distributed CoMP Architecture

However, the cooperating BSs does not share UE data, rather only exchange synchronization information and scheduling information via X2 interface [41]. Therefore, full mesh connectivity among the CoMP set is also mandatory under this architecture. However, though this architecture largely reduced signaling overhead on backhaul and X2 interface but highly depends on radio access link. Also, this architecture is very sensitive to UE's movements and timing mismatch [15].

\section{Performance Evaluations of Comp Transmission}

In this section we represent the performance gain using CoMP transmission technology and compare the result with the traditional network of non-CoMP transmission. The simulation result basically conducted by 3GPP Radio Access Network (working group 1) [47]. It is also noted that the simulation result has been evaluated by using a specific set of assumptions [48], and it may vary depending on the assumptions on, e.g., impairments modeling and scheduler implementation. Also, the evaluation has been performed in both of homogeneous network and heterogeneous network with low power own identity BSs are deployed within a macro-BS coverage area. Figure 5 and 6 shows the relative performance gain of CS-CoMP and JT-CoMP (coherent) implementations compared to Multi-User MIMO (MU-MIMO) implantation in a non-CoMP network. Figure 5 depicts the result in case of homogeneous network, whereas figure 6 presents the heterogeneous network. "Cell tput", "Cell-edge UE tput" and "Macrocell coverage sum tput" depicts the average throughput of the entire cell, the throughput of users in the lower 5 percent-tile throughput distribution of the users and the sum of all cell throughput in the macrocell area in the network, respectively. 


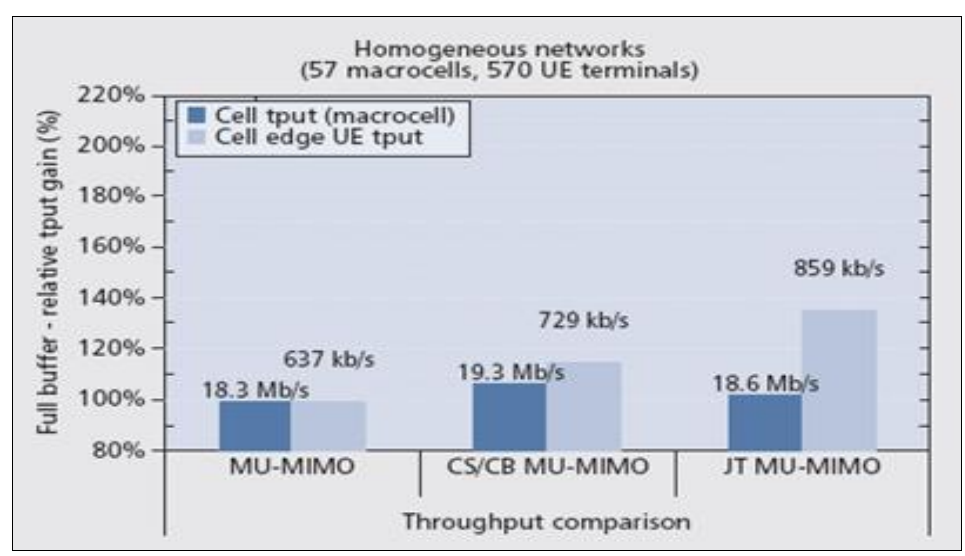

\section{Figure 5. Relative Performance Gain of CoMP Techniques in Homogeneous Network}

In Figure 5, it is seen that by using CoMP transmission better throughput gain is obtained for the cell-side UEs; in JT-CoMP it around 1.35 times more than no-CoMP MU-MIMO and in CS-CoMP the gain factor is around 1.15. However, in heterogeneous networks (figure 6) a high gain is observed in JT-CoMP transmission technology which is 2.15 times more than the non-CoMP network. This is because in heterogeneous networks many pico-cells are deployed within macrocell coverage area which creates more cell boundaries and the overlay of macro and pico-cells with different transmission powers further increase the interference arena. Therefore, more UEs take advantage from CoMP transmission in heterogeneous networks.

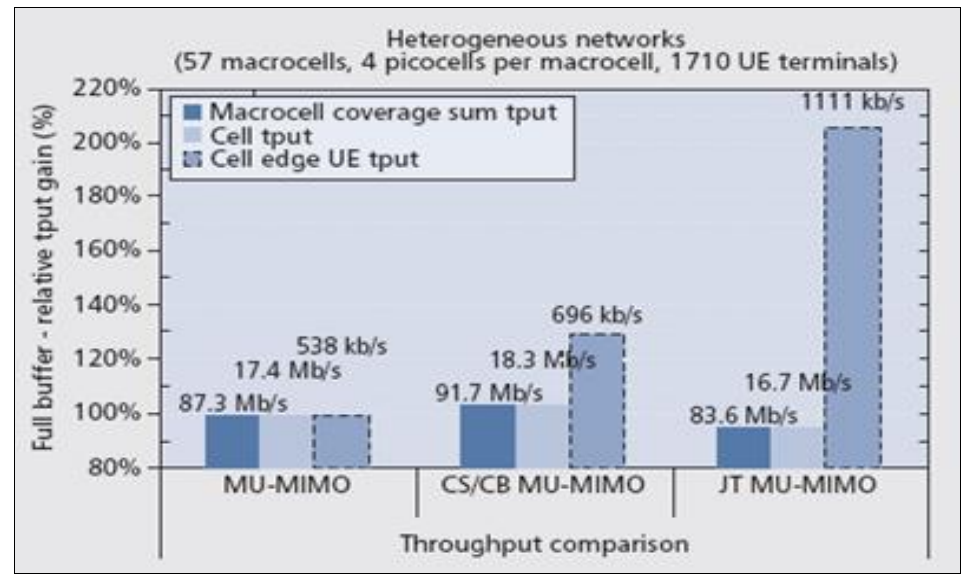

\section{Figure 6. Relative Performance Gain of CoMP Techniques in Heterogeneous Network}

Moreover, in both types of network, JT-CoMP transmission contributes larger performance gains over CS-CoMP, but it requires sharing more information among cooperating BSs which unfortunately results huge signaling impact on backhaul/X2 interfaces. In addition, figure 6 shows that the average cell throughput of JT-CoMP in the heterogeneous network seems to be lower than for the non- CoMP MU-MIMO scenario, but this is due to the fact that different scheduling algorithms were implemented and simulated in JT-CoMP scheme which my affects on overall throughput. 
The above results demonstrate that CoMP techniques are a promising technology for LTEA networks, especially where the cellular deployment is not very regular and uniform but has rather complex cellular boundaries with varying user distributions. CoMP will thereby contribute to providing to the end user a more uniform experience of mobile broadband across the network coverage.

\section{Conclusion}

In this article, we provide an extensive survey on downlink CoMP operation recently proposed for LTE-A radio network standard. We studied the up-to-date CoMP techniques, CoMP architectures and CoMP sets for LTE-A standard from operational perspective. This survey clearly indentifies the benefits and complexities of each CoMP techniques with all possible deployment architectures. The key issue of CoMP implementation in real network is also emphasized. From the discussion it seems that coherent JT-CoMP provides optimal performance but the cost of high signaling overhead, operation complexity, mobility sensitivity and strict synchronization etc. On the other hand, CS-CoMP provides a great release from these challenges but unable to meet desirable throughput for cell edge user. In terms of information sharing among CoMP set BSs fully distributed architecture is most durable but required full mesh reliable connectivity between the BSs.

The performance evaluations show that cooperative transmission techniques have the potential to improve the performance of cellular system, especially for cell-edge UEs throughput gain. However, the performance of the systems is highly depends on the reliability of the control signaling exchange, especially the probability of exchanging CSI. Moreover, the performance of UEs is not considered here which may contribute a great roll to get the fruitful solution from CoMP transmission system.

\section{References}

[1] M. Rumney, "LTE and the Evolution to 4G Wireless: Design and Measurement Challenges", Copyright Agilent Technologies, 2013, Published by John Wiley \& Sons, Ltd.

[2] A. Toskala and H. Holma, "WCDMA for UMTS - HSPA Evolution and LTE", Fourth Edition, John Wiley \& Sons Ltd., (2009).

[3] A. Damnjanovic, J. Montojo, Y. Wei, T. Luo, M. Vajapeyam, T. Yoo, O. Song and D. Malladi, "A Survey on 3GPP Heterogeneous Networks", IEEE Wireless Comm., vol. 18, no. 3, (2011), pp. 10-21.

[4] F. Pantisano, M. Bennis, W. Saad, M. Debbah and M. Latva-aho, "On The Impact of Heterogeneous Backhauls on Coordinated Multipoint Transmission in Femtocell Networks", IEEE International Conference on Communications, Ottawa, (2012) June, pp. 5064-5069.

[5] S. M. Cheng, S. Y. Lien and F. S. Chu, "On Exploiting Cognitive Radio to Mitigate Interference in Macro/Femto Heterogeneous Networks", IEEE Wireless Comm., vol. 18, no. 3, (2011), pp. 40-47.

[6] A. Ghosh, N. Mangalvedhe, R. Ratasuk, B. Mondal, M. Cudak, E. Visotsky, T. Thomas, J. Andrews, P. Xia, H. S. Jo, H. Dhillon and T. Novlan, "Heterogeneous cellular networks: From theory to practice", IEEE Communication Magazine, vol. 500, no. 6, (2012), pp. 54-64.

[7] J. Sangiamwong, Y. Saito, N. Miki, T. Abe, S. Nagata and Y. Okumura, "Investigation on Cell Selection Methods Associated with Inter-Cell Interference Coordination in Heterogeneous Networks For LTEAdvanced Downlink", $11^{\text {th }}$ European Conference on Sustainable Wireless Technologies (IEEE Piscataway), (2011), pp. 1-6.

[8] D. Gesbert, S. Hanly, H. Huang, S. Shamai, O. Simeone and W. Yu, "Multi-cell MIMO Cooperative Networks: A New Look at Interference”, IEEE Journal of Selected areas of Communications, vol. 28, no. 9, (2010), pp. 1380-1408.

[9] 3GPP R1-110461, "Baseline schemes and focus of CoMP studies", Ericson TSG-RANWG1, no. 63, (2011).

[10] 3GPP, "Mobile Broadband Innovation Path to 4G: Release 9, 10 and Beyond", TR, (2010) February.

[11] D. López-Pérez, I. Güvenç, G. Roche, M. Kountouris and T. Quek, J Zhang, "Enhanced Inter-Cell Interference Coordination Challenges in Heterogeneous Networks", IEEE Wireless Communication, vol. 18, no. 3, (2011), pp. 22-30. 
[12] Q. Li, R. Q. Hu, Y. Qian and G. Wu, "Cooperative Communications For Wireless Networks: Techniques and Applications in LTE-Advanced Systems", IEEE Wireless Comm., (2012), pp. 22-29.

[13] R. Irmer, H. Droste, P. Marsch, M. Grieger, G. Fettweis, S. Brueck, H-P. Mayer, L. Thiele and V. Jungnickel, "Coordinated Multipoint: Concepts, Performance, and Field Trial Results", IEEE Communication Magazine, (2011), pp. 102-111.

[14] D. Lee, H. Seo, B. Clerckx, E. Hardouin, D. Mazzarese, S. Nagata and K. Sayana, "Coordinated Multipoint Transmission and Reception in LTE-Advanced: Deployment Scenarios and Operational Challenges", IEEE Communication Magazine, (2012), pp. 148-155.

[15] E. Pateromichelakis, M. Shariat, A. Quddus and Rahim Tafazolli, "On the Evolution of Multi-Cell Scheduling in 3GPP LTE / LTE-A", IEEE Communication Surveys \& Tutorials, (2012).

[16] Z. Mayer, J. Li, A. Papadogiannis and T. Svensson, "On the impact of backhaul channel reliability on cooperative wireless networks", IEEE International Conference on Communications, Budapest, (2013) June, pp. 5284-5289.

[17] J. Zhang, "Networked MIMO with clustered linear precoding", IEEE Wireless Communications, vol. 8, no. 4, (2009), pp. 1910-1921.

[18] M. Vu and A. Paulraj, "MIMO wireless linear precoding”, IEEE Signal Processing Magazine, vol. 24, no. 5, (2007), pp. 86-105

[19] Z. Bai, C. Spiegel, G. Bruck, P. Jung, M. Horvat, J. Berkmann, C. Drewes and B. Gunzelmann, "On the Physical Layer Performance with Rank Indicator Selection in LTE/LTE-Advanced System”, IEEE Workshop on Personal, Indoor \& Mobile Radio Comm., (2010), pp. 393-398.

[20] C. B. Chae, S. Kim and R. Heath, "Network Coordinated Beamforming for Cell-Boundary Users: Linear and Nonlinear Approaches", IEEE J. of Sel. Topics Signal Process, vol. 3, no. 6, (2009), pp. 1094-1105.

[21] P. Hosein, "Cooperative Scheduling of Downlink Beam Transmissions in a Cellular Network", IEEE GLOBECOM Workshops, (2008) December, pp. 1-5.

[22] P. Hosein and C. van Rensburg, "On the Performance of Downlink Beamforming with Synchronized Beam Cycles", $69^{\text {th }}$ IEEE Conference on Vehicular Tech., (2009) April, pp. 1-5.

[23] C. V. Rensburg and P. Hosein, "Interference Coordination Through Network-Synchronized Cyclic Beamforming", $70^{\text {th }}$ IEEE Conference on Vehicular Technology, (2009) September, pp. 1-5.

[24] J. Ellenbeck, M. Hammoud, B. Lazarov and C. Hartmann, "Autonomous Beam Coordination For The Downlink of An Imt-Advanced Cellular System", IEEE European Wireless Conference, (2010), pp. 602607.

[25] M. Vemula, D. Avidor, J. Ling and C. Papadias, "Inter-Cell Coordination, Opportunistic Beamforming and Scheduling”, IEEE Conference on Comm., vol. 12, (2006), pp. 5319-5324.

[26] 3GPP, "Inter-Cell Interference Mitigation through Limited Coordination", Samsung R1-082886, (2008) August.

[27] 3GPP, "CoMP Configurations and UE/eNB Behaviors in LTE-Advanced", LG Electronics R1-090782, (2009) February.

[28] 3GPP, "Multi-cell PMI coordination for downlink CoMP", ETRI R1-091490, (2009) March.

[29] 3GPP, "Aspects of Joint Processing in Downlink CoMP”, Tech. Rep. R1-090193, (2009) January.

[30] K. Lee and D. Williams, "A Space-Time coded Transmitter Diversity Technique for Frequency Selective Fading Channels”, IEEE Workshop on Sensor Array and Multi-channel Signal Processing, (2000), pp. 149152.

[31] M. Feng, X. She, L. Chen and Y. Kishiyama, "Enhanced Dynamic Cell Selection with Muting Scheme for DL CoMP in LTE-A”, IEEE Conference in Vehicular Technology, (2010) May, pp. 1-5.

[32] 3GPP, "Downlink CoMP”, Ericsson R1-090914, (2009) February.

[33] 3GPP, "Setup of CoMP cooperation areas", Nokia-Siemens Networks R1-090725, (2009) February.

[34] A. Papadogiannis, D. Gesbert and E. Hardouin, "A Dynamic Clustering Approach in Wireless Networks with Multi-Cell Cooperative Processing”, IEEE Conference on Communications, (2008) May, pp. 4033-4037.

[35] J. Hoydis, M. Kobayashi and M. Debbah, "On the Optimal Number of Cooperative Base Stations in Network MIMO", CoRR abs/0912.4595, (2009).

[36] A. Barbieri, P. Gaal, S. Geirhofer, T. Ji, D. Malladi, Y. Wei and F. Xue, "Coordinated Downlink Multi-Point Communications in HeterogeneousC Networks", IEEE workshop in Information Theory and Applications, (2012), pp. 7-16.

[37] J. Li, A. Papadogiannis, R. Apelfrojd, T. Svensson and M. Sternad, "Performance Evaluation of Coordinated Multi-Point Transmission Schemes with Predicted CSI", $23^{\text {rd }}$ IEEE Symposium on Personal Indoor and Mobile Radio Communications, Sydney, (2012) September, pp. 1055-1060.

[38] A. Papadogiannis and T. Svensson, "Performance Analysis of Centralized Relay Selection With Unreliable Control Information," IEEE Conference on Vehicular Tech., (2012) September, pp. 1-5.

[39] E. Seidel, "Initial Thoughts on LTE-Advanced for 3GPP Release 10," CTO LTE World Summit, Berlin, (2009) May. 
[40] L. Scalia, T. Biermann, C. Choi, K. Kozu and W. Kellerer, "Power-Efficient Mobile Backhaul Design for Comp Support in Future Wireless Access Systems", IEEE Conference on Computer and Communications Workshops, Shanghai, (2011) April, pp. 253-258.

[41] C. Shen, T. H. Chang, K. Y. Wang, Z. Qiu and C. Y. Chi, "Distributed Robust Multi-Cell Coordinated Beamforming with Imperfect CSI: an ADMM Approach”, IEEE Transaction on Signal Processing, vol. 60, no. 6, (2012), pp. 2988-3003.

[42] O. Simeone, O. Somekh, E. Erkip, H. Poor and S. Shamai, "Robust Communication via Decentralized Processing with Unreliable Backhaul Links", IEEE Transaction on Information Theory, vol. 57, no. 7, (2011), pp. 4187-4201.

[43] R. Fritzsche and G. Fettweis, "CSI Distribution for Joint Processing in Cooperative Cellular Networks", IEEE Vehicular Technology Conference, San Francisco, (2011) September, pp. 1-5.

[44] C. Khirallah, D. Vukobratovic and J. Thompson, "On Energy Efficiency of Joint Transmission Coordinated Multi-Point in LTE-Advanced", IEEE Conf. on WSA, Germany, (2012), pp. 54-61

[45] H. Bang Papadogiannis, D. Gesbertand and E. Hardouin, "Efficient Selective Feedback Design for Multi-cell Cooperative Networks", IEEE Conference on Vehicular Technology, vol. 60, no. 1, (2011), pp. 196-205.

[46] S. Ramprashad, H. Papadopoulos, A. Benjebbour, Y. Kishiyama, N. Jindal and G. Caire, "Cooperative Cellular Networks using Multi-User MIMO: Trade-Offs, Overheads and Interference Control Across Architectures", IEEE Communications Magazine, vol. 49, no. 5, (2011), pp. 70-77.

[47] 3GPP, "Coordinated Multi-Point Operation for LTE”, TSG RAN WG1, (2011) September.

[48] 3GPP, "R1-111628, Phase 1 CoMP Simulation Evaluation Results and Analysis for Full Buffer", LG Electronics TSG RAN WG1, May (2011).

\section{Authors}

Md. Shipon Ali achieved his B.Sc. in Electronics \& Communication Engineering from Khulna University, Bangladesh in 2009. He achieved "University Gold Medal" from Khulna University for securing first position with distinction by obtaining the highest CGPA in Bachelor Examinations among all disciplines under School of Science, Engineering and Technology in the session 2007-2008. Now, he is working as a "senior Engineer" in a multinational telecom operator in Bangladesh. His research interests are dynamic radio resource management, interference management in HetNets, Advanced digital signal processing system and optical communication system. 